Recherches en didactique des langues et des cultures

Les cahiers de l'Acedle

8-1| 2011

Les langues tout au long de la vie: Permanences et évolutions en didactique des langues

\title{
La mémorisation phonologique précoce de l'anglais : un atout pour l'apprentissage tout au long de la vie ?1. Introduction
}

Yvon Rolland

\section{OpenEdition}

\section{Journals}

Édition électronique

URL : http://journals.openedition.org/rdlc/2249

DOI : $10.4000 /$ rdlc. 2249

ISSN : 1958-5772

Éditeur

ACEDLE

Référence électronique

Yvon Rolland, « La mémorisation phonologique précoce de l'anglais : un atout pour l'apprentissage tout au long de la vie ?1. Introduction », Recherches en didactique des langues et des cultures [En ligne], 8-1 | 2011, mis en ligne le 25 août 2017, consulté le 21 avril 2019. URL : http:// journals.openedition.org/rdlc/2249; DOI : 10.4000/rdlc.2249

Ce document a été généré automatiquement le 21 avril 2019

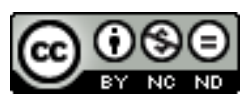

Recherches en didactique des langues et des cultures is licensed under a Creative Commons AttributionNonCommercial-NoDerivatives 4.0 International License 


\title{
La mémorisation phonologique précoce de l'anglais : un atout pour l'apprentissage tout au long de la vie ?1. Introduction
}

\author{
Yvon Rolland
}

1 La mémorisation phonologique serait capitale pour accéder au niveau lexical. Elle relève de repères incluant les phonèmes initiaux et finaux du mot, le nombre de syllabes et le schéma accentuel (Tip of the Tongue phenomenom, Randall, 2007 : 103). Pour autant, la mémoire n'est pas "une simple forme de cognition, mais elle est aussi la forme même de la cognition", nous dit Tiberghien (1983 in Van Der Linden \& Bruyer, 1991 : 9).

P. Roach nous explique que

la phonétique consiste à décrire les sons que nous utilisons en parlant, et lorsque nous évoquons les fonctions des phonèmes dans la langue, les rapports entre phonèmes, ainsi que la dimension abstraite des sons, nous sommes dans le domaine de la phonologie (Roach, $2000: 44$ ).

Nous nous centrons sur l'apprentissage phonologique, et par conséquent la mémorisation, qui commencent à l'école primaire, dans la mesure où la mise en place d'un processus efficace nous semble importante pour l'apprenant dans la perspective de l'apprentissage tout au long de la vie. En effet, une mémorisation phonologique effective va faciliter la construction du domaine linguistique en langue, mais aussi dans des disciplines non linguistiques si l'apprenant est amené à suivre des cursus où l'anglais est langue d'enseignement. Ce processus de mémorisation phonologique impliquant l'articulation et la réalisation des phonèmes, la maittrise du rythme et de l'intonation peut se faire dès les années du primaire, afin de faciliter le cursus du collège et du lycée, de l'enseignement supérieur (échanges Erasmus) comme lors de stages en entreprise à l'étranger. Nous allons ainsi nous focaliser sur les stratégies d'apprentissage facilitant la mémorisation auprès d'un public spécifique, celui de jeunes apprenants. 
4 La recherche-action que nous proposons est qualitative, descriptive, analytique, mais aussi expérimentale. Son objectif est d'essayer de comprendre la mémorisation et d'améliorer son efficacité dans le cadre de l'appropriation phonologique, importante dans l'enseignement primaire et pouvant servir à la formation à plus long terme, à l'adolescence et à l'âge adulte.

5 Nous analyserons la complexité du processus de mémorisation, les atouts et obstacles liés à l'âge, ainsi qu'une réalité institutionnelle qui annoncent certaines limites.

6 Nous tenterons d'apporter un éclairage sur la mémorisation phonologique, l'apport des nouvelles technologies et de nouveaux processus humanistes à définir, ayant pour but de faciliter la maîtrise phonologique à long terme (Van Der Linden \& Bruyer 1991, Lieury, 1997, Randall, 2007). Par humanisme, nous entendons la prise en compte de l'homme et des valeurs humaines.

7 Nous présenterons le contexte de notre recherche en partant d'un test diagnostic, donné dans une classe primaire, portant sur les phonèmes, le rythme et l'intonation. Ce test dévoile les limites de la mémorisation phonologique chez les apprenants. Enfin, nous proposerons une séquence qui va intégrer la mémorisation phonologique, et nous terminerons par un bilan de cette étude.

\section{Le cadre scientifique}

\subsection{La complexité de la mémorisation phonologique}

8 Nous pouvons nous demander si la mémoire relève de représentations mnésiques qui sont stockées dans un espace structurellement unitaire, selon un modèle informatique, ou s'il faut envisager des neurones en réseau, dont les informations sortantes rétroagissent sur l'ensemble du réseau, selon un modèle connexionniste : en d'autres termes, si la mémoire est localisée ou distribuée (Tiberghien, 1991 : 22-32).

\subsubsection{Modèles néo-informatiques}

9 Les modèles néo-informatiques relèvent d'un système de traitement de l'information composé de modules de traitement élémentaires organisés de façon séquentielle. Selon Atkinson et Shiffrin (1968), les processus de contrôle seraient très proches du fonctionnement d'un ordinateur, avec sa mémoire centrale et son unité de contrôle. Le problème est de savoir quelle est la relation entre la mémoire sémantique et la mémoire épisodique, toutes deux mémoires à long terme, l'une orientée vers le sens, l'autre vers l'affect. Selon Tulving (1983), l'accès aux représentations se ferait par l'inférence pour le processus sémantique et par le contexte pour le processus épisodique. Le modèle d'Andersen (1983) évoque quant à lui une mémoire de travail opérationnelle et transitoire en relation constante avec une mémoire permanente de type déclaratif et une autre permanente aussi, de type procédural.

\subsubsection{Modèles neuro-mimétiques ou néo-connexionnistes}

10 Ces modèles s'opposent radicalement aux précédents et partent du principe que la mémoire peut être distribuée, délocalisée. L'information entre dans un neurone qui est ainsi modélisé et qui va ensuite rétroagir sur l'ensemble des autres neurones avec un 
système bouclé sur lui-même. Ce qui est mémorisé n'est plus localisable précisément comme précédemment, mais se trouve dans chaque partie du réseau neuro-mimétique. Les possibilités de récupération après une atteinte lésionnelle du cerveau sont connues et tendraient à prouver l'existence de ces mémoires distribuées. L'existence de ces boucles de rétroaction a été aussi mise en évidence. Il existerait peut-être une spécialisation des différentes structures cérébrales, ce qui relativiserait, au moins en partie, l'idée selon laquelle tout est connecté avec tout (Van Der Linden \& Bruyer, 1991).

\subsubsection{Modèles néo-holographiques}

11 Les modèles néo-holographiques occupent une position intermédiaire par rapport aux modèles préalablement cités, et partent du principe que la distribution de l'information se fait dans la totalité de l'espace mnésique. Alors que les architectures néoconnexionnistes associent cette distribution à la structure même des neurones, les modèles néo-holographiques la limitent au concept de trace (Eich, 1982, in Tiberghien, 1991 : 33). Ces deux modèles rejettent la métaphore spatiale, mais les modèles néoholographiques conservent le point de vue fonctionnel et modulaire des modèles néoinformatiques. Nous le voyons, ces multiples modèles ne peuvent pas clarifier la compréhension du processus mnésique.

\subsubsection{Phases mnésiques}

Selon les modèles néo-informatiques, la mémorisation reposerait sur plusieurs phases.

- La mémoire immédiate serait éphémère et ne peut porter que sur quelques éléments seulement. La mémorisation ne se fait pas correctement si la perception est insuffisante, la saisie de l'information est trop rapide, incomplète ou floue.

- La mémoire à court terme serait de quelques minutes. Elle dépend beaucoup du contexte de l'apprentissage, de l'intérêt ressenti, c'est à dire de l'affectif.

- Enfin, la mémoire à long terme relève de l'aboutissement d'un processus réussi. Elle ne peut intervenir que si l'information a été connectée et que l'enregistrement s'est effectué sur des repères permettant la récupération. Un blocage émotionnel ou un manque d'intérêt, plus simplement, des répétitions décontextualisées, peuvent enrayer le mouvement et perturber l'apprentissage à long terme.

\subsubsection{Processus de mémorisation}

Les étapes du processus de mémorisation sont multiples.

L'encodage désigne la modification des informations sensorielles en représentations mentales, véritables entrées de notre mémoire. Les processus d'encodage sont un véritable filtre contrôlé inconsciemment par nos habitudes, nos motivations, nos projets et nos conduites. Les informations perceptives à encoder (visuelles, auditives, etc.) sont multiples, de même que leur signification (mot, phrase, texte), leur organisation.

La durée de l'exposition joue aussi. L'information encodée ne peut survivre sans les mécanismes de la consolidation, indispensables au maintien en mémoire permanente. L'activation de certaines structures cérébrales est une condition importante de cette consolidation (Van Der Linden \& Bruyer, 1991: 10). Une fois encodée et consolidée, l'information mnésique sera disponible en mémoire permanente. Le stade ultime est celui 
de la récupération qui se fait grâce à des clés d'accès que sont les indices directs ou indirects (questions explicites, contexte général).

Il est intéressant de noter que les étapes que sont l'encodage, le stockage, le rappel et la reconnaissance sont intimement liées au processus d'apprentissage. Les désordres de l'une vont toujours de pair avec les désordres de l'autre (Trocmé-Fabre, 1992 : 74).

\subsubsection{Phénomène de l'oubli}

17 Tulving (1983, in Van Der Linden \& Bruyer, 1991 : 12) précise que l'accès à un souvenir est d'autant plus facile que les conditions contextuelles de sa récupération ressemblent aux conditions contextuelles de sa constitution, de sa mise en mémoire ou de son encodage. Ainsi nous oublions des informations dont la qualité d'encodage a été insuffisante, nous oublions celles correctement codées mais inhibées parce que les indices de récupération sont inappropriés. L'absence de similitudes ou de compatibilités sémantiques entre les conditions contextuelles d'élaboration de la trace mnésique et les conditions contextuelles de sa récupération seraient l'une des causes fondamentales de l'oubli. Sémantisme et contexte semblent être à la base du problème. Ceci pose le problème de la décontextualisation ou transfert, qui devrait sans doute être assez proche du contexte initial. Sans ces précautions prises lors de l'apprentissage précoce et plus tard dans la récupération, la possibilité de mémoriser pour l'apprenant semble gênée. La multiplicité de ces approches ne facilite pas l'analyse théorique de la mémorisation. Le contexte institutionnel est un autre obstacle, comme nous allons le voir.

\subsubsection{Une réalité institutionnelle française peu facilitatrice}

18 Les méthodologies structurales audio-orales et audio-visuelles qui ont prévalu en France mettaient déjà en avant l'apprentissage associatif, mais en préconisant la mémorisation du par cœur. Elles étaient axées sur un formalisme souvent dénué de contextualisation significative et de communication (Randall, 2007 : 148).

L'avènement de l'approche communicative de première génération (Bailly, 1998 : 40-41) a tenté de mettre en avant des situations de communication concrètes. L'obédience était pragmatique. La priorité revenait de fait à l'utilisation de données fonctionnelles en insistant sur le sens, mais en négligeant la forme et la mémorisation.

L'approche communicative de deuxième génération (Bailly, 1998 : 41) a tenté d'intégrer la dimension cognitiviste, mais en négligeant les dimensions motivationnelle et phonologique. La dimension phonétique et phonologique bien que présente, pourtant, dans les textes officiels (phonèmes, rythme et intonation - 1995), se trouvait souvent atomisée dans les manuels sous la forme d'annexes situées dans des encarts détachés des activités centrales de réception et de production. Par ailleurs, la dimension réflexive mise à l'honneur par la pratique raisonnée de la langue et d'obédience énonciative, est davantage mise en pratique pour la morphosyntaxe que pour la phonétique ou la phonologie.

21 La perspective actionnelle et l'approche par les compétences (Beacco, 2007) tentent d'intégrer les savoirs phonologiques afin d'éviter cette atomisation préjudiciable. L'approche par les compétences présente pourtant le risque de rester à un apprentissage procédural spécifique et de ne pas intégrer la dimension déclarative propre à la phonologie. Si cette constante résurgence phonologique est de mise dans les orientations 
institutionnelles françaises depuis des années (Instructions officielles de 85, 95, 2005), force est de constater que sa mise en place s'avère délicate.

Les nouvelles technologies sont recommandées, elles aussi, depuis longtemps par l'institution. Elles apporteraient souvent une approche co-constructive, l'enseignant étant davantage considéré comme une aide que comme une autorité. Cette perception de l'apprentissage est salutaire, car mieux acceptée. Mais leur intégration maladroite peut engendrer de nouveaux problèmes. Les machines informatiques imposent de plus en plus leurs modèles d'intelligence artificielle. Le danger serait effectivement "d'ignorer tout de la genèse pré-conceptuelle et des bases sensori-motrices, psycho-affectives" (Linard, 1996 : 26).

Il reste qu'une composante humaniste intégrant la dimension mentale, mnésique, émotionnelle et comportementale complèterait utilement, dans la perspective d'un apprentissage à long terme, des fondements de référence linguistiques, cognitivistes, didactiques et technologiques cloisonnant trop forme et sens, phonétique et phonologie, apprentissage et mémorisation. Ceci nous amène à ce que disent Van der Linden et Bruyer :

l'incapacité à se rappeler une information provient toujours du fait que l'on se trouve dans des conditions situationnelles, cognitives et affectives beaucoup trop éloignées de celles qui ont présidé à la constitution du souvenir (Van der Linden et Bruyer,1991 : 12).

\subsection{L'apport de nouveaux processus didactiques humanistes}

De nouveaux concepts humanistes liés à la neurobiologie, la psychologie sociale, l'éthologie vont permettre de transcender le spectre théorique traditionnel.

\subsubsection{L'âge : atouts et obstacles}

D. Singleton (1989) analyse le problème de l'âge critique de réceptivité. Les positions divergent sur ce point entre ceux qui pensent que la période critique est close dès deux ans et ceux qui prétendent que les adultes sont toujours à même d'apprendre. Tout dépend en fait du domaine d'apprentissage linguistique, phonologique ou morphosyntaxique. Il faut aussi distinguer l'apprentissage de la L1 et celui de la L2. L'apprentissage de la L2 est influencé par celui de la L1, notamment sur le plan phonologique.

Reprenant les travaux de Cummins (in Singleton, 1989: 113), D. Singleton explique également que l'apprentissage concerne le domaine de la communication (Basic Interpersonal Communication Skills) comme celui du domaine cognitif (Cognitive Academic Language Proficiency). Se pose ainsi le problème de l'âge vu tantôt comme un atout, tantôt comme un obstacle.

D.Singleton (1989 : 101-117) énumère les quatre positions possibles :

- La première vise à privilégier l'apprentissage précoce pour le volet phonologique, arguant $\mathrm{du}$ fait que les jeunes élèves ont une plasticité auditive, et que vers douze ou quinze ans, l'acquisition serait gênée par la phonologie de la L1;

- La deuxième part du principe d'un apprentissage favorisé avec l'âge, des adolescents apprendraient autant en cinq ans que d'autres plus jeunes en onze. Mais en fait, il s'agit surtout de la syntaxe et du domaine de conceptualisation morpho-syntaxique2017-08-25T15:28:00AS ; 
- La troisième met en avant l'apprentissage très précoce pour la compétence phonologique plus que pour la syntaxe ;

- La quatrième insiste sur l'atout que constitue l'apprentissage précoce à très long terme. processus de décodage phonologique, lexical et grammatical. Ceci enclenche un processeur phonétique acoustique menant à une représentation phonétique, puis prosodique. Les opérations mentales liées au passage de l'input à l'output sur le plan phono-articulatoire, montrent à l'évidence, une nativisation des formes et du sens inéluctables (Narcy-Combes, 2005 : 107), c'est à dire une analyse par l'apprenant de toute nouvelle donnée en L2 selon les critères mémorisés de L1 (Andersen, 1983 : 11), ce que Jean Piaget définissait comme l'assimilation. La perception phonologique de la langue cible nous amène à des représentations contre-productives selon Cyrulnik :

l'observation la plus banale devient un prodigieux travail de création neuroimaginaire ; toute perception est conditionnée par qui nous sommes, et la façon dont nous ressentons le contexte dans lequel elle se situe (Cyrulnik, 1983 : 65). l'expérience antérieure façonnent inconsciemment nos représentations. L'étude du cerveau montre qu'aucune partie ne fonctionne isolément (Trocmé-Fabre, 1992 : 213). Le cerveau primitif ou reptilien, très attaché aux habitudes et aux automatismes est un des moteurs mnésiques de la nativisation. Il faudrait sans doute rééquilibrer la dimension mnésique par une contextualisation de l'apprentissage facilitée par les nouvelles technologies, avec la présence de supports visuels et sonores plus aboutis, et une participation plus active et constructive de l'apprenant.

\subsubsection{Accommodation phonologique}

L'output relève de processus d'encodage grammaticaux, lexicaux, phonologiques, puis phonético-articulatoires. Le mécanisme fonctionnera d'autant mieux que les données seront comprises, et mémorisées sur le plan cognitif, ce que l'on nomme "intake" (Narcy in Ginet, 1997 : 53). L'apprenant doit en fait entrer en conflit avec sa vision du monde ce qui s'accompagne d'une déstructuration cognitive et affective (Develay, 1993). Il faut donc faciliter les représentations phonologiques de l'apprenant en intégrant des repères phonologiques connus pour faciliter la cognition générale et le processus de mémorisation phonologique des éléments linguistiques nouveaux. Le processeur phonologique est en rapport avec le processeur sémantique et le contexte (Randall, 2007 : 75). Ceci permettra à l'élève de mieux appréhender certains décalages afin d'atteindre un début de dénativisation (Andersen, 1983) ou d'accommodation (Piaget) de l'interlangue ce qui la rendra plus conforme aux normes de la langue cible. La dénativisation fait suite à la nativisation: véritables forces antagonistes, elles relèvent d'un processus de cognition 
générale et sont partie intégrante de la mémorisation, comme nous le rappellent Andersen (1983), citant Jean Piaget, et Tiberghien (1983 in Van Der Linden \& Bruyer, 1991).

\subsubsection{Mémoire de travail}

Van Der Linden \& Bruyer (1991: 22) privilégient la conception néo-informatique modulaire de la mémoire dans la mesure où elle intègre la mémoire de travail et ses multiples composants à court terme et la mémoire à long terme et ses constituants que sont la mémoire visuelle, verbale, la mémoire épisodique ou affective, sémantique et procédurale. Il se réfère au modèle de mémoire de travail de Baddeley (1992) "désignant le stockage temporaire d'informations en vue de réaliser des tâches cognitives diverses, comme la compréhension du langage" (Buser, 1998 :171). Les parties cérébrales impliquées sont les lobes temporaux, pariétaux et frontaux. Un administrateur central à capacité limitée agit sur un système visuo-spatial, un système phonologique et la récapitulation articulatoire (Van Der Linden \& Bruyer, 1991: 114). N.C. Ellis (2001: 36) insiste sur la dimension auditive et visuelle du processus mnésique de travail. P. Buser parle d'un mode opératoire explicite (1998: 182).

Mais, paradoxalement, M. Randall précise l'obédience psychologique connexionniste de cette mémoire de travail qui relève de concepts d'apprentissage associatifs (2007: 27). Il explicite le rôle central de la mémoire de travail qui, dans le cadre de la compréhension de l'oral, reçoit les indices des canaux sensoriels ainsi que des segments phonétiques de la mémoire dite "échoïque" (op. cit. : 92). C'est par la répétition et l'apprentissage associatif que la mémoire de travail transfère les données vers la mémoire à long terme (op. cit. : 134).

Van Der Linden \& Bruyer $(1991: 116)$ précisent que la mémoire de travail à court terme ne correspond pas à un système unitaire mais à un ensemble de composantes multiples distinctes. Elle se trouve également influencée aussi par la mémoire à long terme qui inclut la mémoire sémantique et ses volets phonologiques, syntaxiques et lexicaux, ainsi que la mémoire épisodique. La mémoire à long terme serait aussi fractionnée entre mémoire sémantique, mémoire épisodique (Tulving, 1972), mémoire déclarative et mémoire procédurale (Squire, 1982), mémoire implicite et mémoire explicite (Schacter, 1987).

Nous le voyons, la mémoire relève de fractionnements et de composantes multiples qui rendent l'analyse délicate. Le mode spatio-temporel interactif propre à un cédérom remplace, d'autre part, le principe logico-syntaxique et linéaire d'une programmation classique. Il est enfin remarquable de constater que la reproduction, la boucle, l'imitation se retrouvent à la fois en linguistique, en psychologie cognitive et en informatique (Linard, 1996 : 96).

\subsubsection{Ancrage perceptif et moteur}

P. Buser (1998: 172) surmonte la complexité des modèles mnésiques pré-cités, en insistant sur l'ancrage de la perception et de l'action aux contenus de la mémoire. Se basant sur les travaux de Fuster (1995), il stipule qu'il faille simplifier la dichotomie des classes de mémoire et se limiter à la distinction entre une mémoire perceptive et une mémoire motrice. La première serait déclarative, la seconde procédurale. La première 
dépendrait des territoires néocorticaux sensoriels associatifs et la seconde d'aires frontales et préfrontales.

L'ancrage mnésique perceptif et moteur pourrait être matérialisé par l'apport d'un cédérom à forte dimension ludique, et donc motivationnelle qui semble intéressant pour dynamiser un contexte d'apprentissage peu satisfaisant. Il comportera des jeux interactifs stimulant la curiosité et l'engagement de l'apprenant. Encore faut-il éviter d'en faire un instrument de maitrise totale, qui replacerait l'enseignant en quelque sorte, mais l'utiliser plutôt comme un partenaire : l'enseignant garde le contrôle de l'introduction des éléments phonologiques, par exemple, et se ferait aider par la machine, qui serait utilisée en plus. L'encodage verbal et imagé est complété par le logiciel, qui concrétise la situation de communication. Nous avons l'aspect ludique, le recours au multisensoriel des matériaux et des activités sensori-motrices proposées (dimension auditive, visuelle et aussi motrice, Dörnyei, 2001: 141). La participation active que cela implique chez les enfants, par l'interaction orale mise en place (entre l'élève et l'ordinateur) et qui serait à la base de toute stratégie motivationnelle (Dörnyei, 2001 : 138), nous amène au principe piagétien des connaissances qui dérivent de l'action.

\subsubsection{Prise en compte de l'affect}

G. Tiberghien avoue aussi les limites de modèles fondamentalement antagonistes de la mémoire. Il préconise l'intégration de l'affect - sans doute une forme de connaissance - dans les modèles de la mémoire humaine et dans la psychologie cognitive (in Van Der Linden \& Bruyer, 1991 : 35).

L'affect du public concerné, les enfants du cycle 3 de l'école primaire, se caractérise par un développement du cerveau faisant la part belle à la partie émotionnelle du cerveau limbique : une myriade de racines se multiplie vers toutes les parties du néo-cortex, "ce qui donne à l'affectivité et aux émotions le pouvoir d'influer sur tout le cerveau et même les centres de la pensée" (Goleman, 1997 : 28). "Le cognitif peut être géré ou contrôlé par l'affectif" (Buser, 1998: 298). Selon D. Goleman, "l'amygdale, complément de l'hippocampe dans le cerveau limbique, véritable alarme chimique, lorsqu'elle est excitée, a pour effet d'imprimer dans la mémoire les moments d'émotion avec une force inhabituelle" (Goleman, 1997 : 39). Nous devons ajouter l'impact de l'affect sur la motivation, car "l'activité est effectuée pour la satisfaction qu'elle apporte" (Thill, 1997 : 377).

41 L'immaturité cognitive relative des jeunes écoliers provient aussi du lent développement progressif du néo-cortex lié au raisonnement. Le cerveau limbique émotionnel, source de mémorisation constante et puissante de l'affectivité doit être prise en considération. L'utilisation d'un cédérom interactif aux activités ludiques et attractives devrait y contribuer.

\subsubsection{Rôle du système sensori-moteur}

La théorie du crible phonologique de Troubetskoy (1986: 54) explique que les sons d'une langue maternelle relèvent de systèmes activés dès l'apprentissage de la parole qui vont déterminer les facultés perceptives des natifs d'une langue donnée et parallèlement, bloquent les sons étrangers. Solliciter le seul canal auditif nous amène à une forme de surdité. Comme nous l'avons souligné, la mémoire échoïque de type auditif qui ne concerne que la forme phonologique n'est viable qu'avec la mémoire de travail, elle-même influencée par la mémoire sémantique et la mémoire épisodique. L'accès au 
sens peut-être facilité par d'autres canaux sensoriels comme le visuel et le kinésique, faisant du registre sensoriel un atout pour la boucle phonologique et la mémoire de travail. H. Trocmé-Fabre (1992: 79) précise que "toute sensorialité en déclenche une autre, grâce au lien établi par l'affectivité". La mémoire imagée serait très puissante (Lieury, 1997 : 39-Ellis, 2001 : 129), de même que la mémoire corporelle. "Une fois que le mot et le geste sont associés, il est possible de retrouver le mot en refaisant le geste : les gestes seraient la réplique de la mémoire verbale" (Williams, $1986: 158)$ ). C. O’Neil (1993: 205) rapporte ce principe: "les mécanismes de l'action s'exercent avant ceux de la réflexion, la situation est représentée par le geste, ce qui leur permet de s'approprier la langue" (Wallon, 1968). "La langue synchronisée avec des réactions physiques permet de mieux comprendre et donc de mieux mémoriser" (Asher-Price, 1967). Placer par exemple ses doigts sur sa gorge pour sentir une vibration (ou pas) permettra de mesurer la différence entre consonnes dentales fricatives sourdes (comme dans "cloth") ou sonores (comme dans "clothes") fera prendre conscience de l'existence des cordes vocales et favorisera l'accommodation phonologique, c'est à dire, rappelons-le, l'adaptation de notre monde interne aux données externes. La kinésie englobe aussi l'émotionnel: "elle concerne les expressions corporelles, posturales, tactiles, motrices et émotionnelles" (Trocmé-Fabre, 1992 : 78). Les cédéroms peuvent aider la multisensorialité, dans la mesure où les canaux visuels, auditifs et moteurs sont sollicités.

\subsubsection{Processus associatifs} l'utilisation du langage et qui véhiculent des idées mentales mémorisées et ancrées dans chacun d'entre nous. ayant un mode opératoire implicite. Il analyse en fait la mémoire implicite qui comprendrait la mémoire procédurale et la mémoire sémantique. Un mot nouveau introduit en phase communicative par un jeu de devinettes sera, par exemple, associé à une affichette et au mime. Les apprenants seront ensuite invités à reproduire le mime afin d'intégrer la langue par le corps. La dimension phonologique sera intégrée en phase d'apprentissage par une association d'affichettes (six pigs, a sad cat, ten pens, Henry the happy hippo), un geste de l'enseignant repris par les enfants pour le rythme (par exemple un claquement des doigts sur la syllabe accentuée) et les schémas intonatifs (faire se lever la classe pour un schéma montant et s'asseoir pour un schéma descendant). Des activités de discrimination phonétique proches des paires minimales (Randall, 2007:52), des phases de vocalisation nécessaires à la mémorisation (Lieury, $1997: 22$ ), des moments de reproduction (Randall, $2007: 127$ ) associés à des aides visuelles et motrices, proposés par un cédérom approprié, seraient salutaires et indispensables. Craig et Lockart (in Van Der Linden, 1991 : 145) proposent, eux aussi, d'intégrer la dimension phonologique au processus de mémorisation épisodique par l'association et les rimes phonétiques. Selon Tulving (1983, in Van Der Linden, 1991 : 12), l'encodage est la meilleure clé d'accès au souvenir par une ré-instauration des conditions situationnelles, affectives et cognitives spécifiques de cet encodage.

\subsubsection{Mémoire épisodique}

Cette dernière conforte le volet de l'affect. Elle concerne des évènements personnellement vécus et qui peuvent être localisés dans l'espace et le temps d'une 
destinée singulière (Tulving, 1983 in Van Der Linden, 1991: 15). Elle serait explicite (Buser, 1998: 182). Les processus associatifs favorisent la mémoire épisodique qui renforce la mémoire de travail selon le modèle décrit par Baddeley (2000). Cette mémoire épisodique est celle du vécu avec sa forte coloration affective. H. Laborit (1996: 88 et 130) insiste sur l'importance de l'affectivité du système limbique cérébral, car "dès qu'il y a sentiment, il y a mémoire et apprentissage", et aussi parce qu'il n'y aurait "pas d'affectivité sans mémoire". Baddeley précise que la mémoire épisodique n'est pas uniquement une forme de mémoire à long terme, et qu'un tampon épisodique agit dès le moyen terme au même titre que le système visuo-spatial et phonologique de la mémoire de travail.

Ceci accrédite l'importance de la mémoire épisodique qui joue un rôle crucial dans le processus mnésique global et dans la mémorisation à long terme. Le processus d'appropriation phonologique ne serait-il pas ainsi favorisé par l'utilisation d'un cédérom qui contribuerait à renforcer la dimension mnésique de façon déterminante?

\section{Mise en œuvre de l'expérimentation}

Le contexte est celui d'une classe de CM2 en troisième année d'apprentissage de l'anglais (CM2 École de Ruisseau Blanc, La Montagne, Circonscription de St Denis 5, Académie de la Réunion). La majorité des enfants a 10 ans. Le contexte institutionnel est français avec la particularité que l'anglais est L3, le français L2, puisque le créole est L1 pour la majorité des élèves. L'enseignante est Professeur d'école habilitée pour l'anglais et titulaire d'un CAFIPEMF (Certificat d'aptitude aux fonctions d'instituteur et professeur d'école maître formateur) option anglais. Le groupe comprend 20 élèves. Selon l'enseignante, ces enfants sont assez inhibés et introvertis, et doivent être mis en confiance pour qu'ils participent.

\subsection{Test diagnostic}

48 L'enseignante et moi-même avons effectué un test diagnostic sur le plan phonologique lors d'une présentation des enfants à tour de rôle devant le groupe classe, mettant en œuvre la production orale en continu et permettant aux élèves de se saluer, de donner leur nom, prénom, âge, adresse et l'humeur du moment ("I'm happy, fine, tired, sleepy etc... "). Les trois critères retenus sont dans les programmes institutionnels français relatifs à la phonologie au primaire, soit "reproduire de manière intelligible les sons, les rythmes et les courbes intonatives" (B.0. 8 du 30 août $07: 7$ ). Les élèves parlent à tour de rôle devant le groupe-classe et doivent réaliser les phonèmes, respecter le rythme et les schémas intonatifs : chaque apprenant est réparti dans des groupes selon les critères établis (la réalisation de phonèmes, la maitrise du rythme et de l'intonation) et le système de mesure. Nous nous sommes inspirés de l'échelle de Harris (Tagliante, $2005: 200$ ) qui oscille de "inintelligible-1, très peu compréhensible-2, peu compréhensible-3, intelligible-4 et très intelligible- 5 ". Le but est de classer les apprenants par critère dans cette échelle. Les résultats sont les suivants :

- Les sons ne sont pas bien acquis. 15 enfants se situent dans les trois niveaux relevant de l'inintelligibilité.

- Pour le rythme, on en dénombre16.

- L'intonation pose problème à 18 enfants. 


\subsection{Proposition de séquence}

\subsubsection{Objectifs} les laisser découvrir la scène initiale, puis de les laisser la revoir p dimension visuelle et auditive est impliquée, puisque le cédérom dévoile un salon et la bande son des échanges. L'enseignante va alors faire arrêter les machines. Il s'agit de faire commenter la situation (Oh no! look! look at the window! what's the problem? a man, a burglar came and took/stole the music instruments! who is it? you? you? They saw the man and they can describe him!).

\subsubsection{Décodage phonologique et encodage du lexique}

L'enseignante laisse les enfants visionner à volonté la phase introductive du cédérom. Un portrait-robot lacunaire apparaît. Puis l'enseignante va projeter la scène sur grand écran avec un vidéoprojecteur. Le portrait-robot lacunaire réapparaît avec un arrêt sur image. Les étapes d'apprentissage lexical impliquent la mémorisation phonologique :

- association de l'affichette réponse et du mot entendu ;

- association de l'affichette, du mot entendu et du geste effectué pour une mémoire immédiate ;

- association de l'affichette, du mot entendu, de rimes phonétiques et articulation en silence des phonèmes des mots nouveaux pour une mémoire de travail ("-look and listen, listen and do: Wow !/AU / a mouth! /T/ thanks! look!/U/ a moustache! a star's moustache!/A :/ a moustache or a shoe?/S/ a nose? no! /U / a nose or a zoo?/z/ eyes! /aI/ five eyes! Hi! this is his hair! /h/ Henry's hair on his chair! /e'/". Puis des paires minimales sont proposées: "listen and repeat the odd man out: wow/mouth/no-oh/how/nose-eyes/five/car-chair/hair/here-hi/oh/how"). Pour chaque phonème intrus, une affichette est placée au tableau: elle illustre visuellement l'articulation du phonème (Baker, $1994:$ 84, 12, 74, 43, 91, 86, 6, 105, 46, 72).

- association du mot entendu, du geste et de la reproduction collective, puis individuelle pour une mémoire de travail ("look, touch and repeat : a mouth, a moustache, a nose, eyes, hair").

L'activité suivante porte toujours sur la mémoire à court terme avec un intérêt ludique certain et une dimension affective, grâce à un jeu (Chinese whispers): l'enseignante désigne des groupes d'enfants qui devront par équipes, le plus rapidement possible, transmettre par vocalisation les éléments phonologiques des mots nouveaux. 


\subsubsection{Décodage et encodage phonologique de la syntaxe} exemple produit: "He's got long black hair. He's got blue eyes. He's got glasses. He's got a big nose. He's got a big mouth. He's got a black moustache". 
61 Les sons semblent mieux maîtrisés (12 élèves relèvent des deux niveaux d'intelligibilité contre 5 lors du premier test) : les voyelles brèves et longues, les consonnes (la fricative non sibilante glottale $/ \mathrm{h} /$, la fricative non sibilante apico-dentale non voisée $(\mathrm{T})$ sont produites correctement, ce qui n'était pas le cas lors du test diagnostic.

62 Le rythme révèle aussi des progrès tangibles, avec un respect des formes accentuées pour 13 enfants, contre 4 seulement au début.

De même l'intonation descendante des déclaratives est améliorée et s'avère positive pour 14 enfants, contre 2 initialement.

Il en résulte qu'une majorité d'enfants relèvent cette fois des deux niveaux d'intelligibilité.

\subsection{Bilan, principes et pistes}

65 Nous avons tenté de respecter les étapes du processus de mémorisation que sont l'encodage et la consolidation phonologique afin de faciliter le stade de la récupération. La phase d'encodage s'est effectuée en tenant compte de la multiplicité des données perceptives, du degré de concentration des apprenants et d'un temps d'exposition suffisant. Nous avons prévu des phases de consolidation.

Pour éviter l'oubli, nous avons favorisé sémantisme et contexte. Nous avons fait en sorte de remettre l'apprenant dans des conditions situationnelles, cognitives et affectives proches de celles qui ont présidé à la constitution du souvenir (Van Der Linden \& Bruyer, $1991: 12)$.

67 Le travail sur les phonèmes s'est effectué avec les technologies cognitives, mais sans renier les conditions qui font l'expérience, à savoir le corporel, le psycho-affectif et le relationnel (Linard, 1996: 19). La part dévolue à l'enseignante a été respectée pour justement équilibrer la dimension humaine. Mais la dimension ludique et motivationelle du cédérom a judicieusement complété le volet psycho-affectif.

Nous avons insisté sur la mémoire de travail, la répétition et la mémoire "échoïque", l'association et la contextualisation nécessaires à un transfert adéquat des données vers une mémoire sémantique et épisodique.

Nous avons tenu compte de l'affect, ainsi que des formes complémentaires de sensorialité (Trocmé-Fabre, 1992: 79) : les déficiences auditives recensées sont ainsi compensées par l'apport de la mémoire imagée et la mémoire corporelle, elle-même émotionnelle. Les processus associatifs ont contribué à cette osmose salutaire.

70 Le bilan, positif, doit être relativisé. Il faut admettre que cette séquence est ponctuelle et qu'une généralisation de cette expérience au sein d'une école, voire d'une circonscription serait intéressante pour mieux valider nos hypothèses et confirmer davantage nos conclusions. Il faudrait aussi suivre certains de ces apprenants sur le long terme afin de mesurer les atouts que cette approche présente dans un apprentissage continué tout au long de la vie, en termes de performance linguistique, non seulement en anglais, 
discipline linguistique, mais aussi par l'anglais dans le cadre des disciplines non linguistiques.

71 Il ressort certains principes-clés qui semblent cependant incontournables pour une meilleure mémorisation phonologique à long terme :

- tout d'abord, il faut contextualiser la situation d'apprentissage et l'utilisation d'un cédérom est à ce sujet particulièrement intéressante, car l'interaction suscitée relève d'une stratégie motivationnelle salutaire.

- il faut judicieusement décoder et encoder les éléments phonologiques en contexte en privilégiant la composante humaniste intégrant les dimensions émotionnelle, comportementale, mentale, cognitive et mnésique.

- il faut méthodiquement consolider la dimension phonologique de façon ludique par des activités adaptées bien contextualisées.

Notre processus de mémorisation phonologique a ainsi privilégié les similitudes et compatibilités sémantiques entre les conditions contextuelles de l'élaboration de la trace mnésique et les conditions contextuelles de sa récupération. Les résultats au test d'accommodation phonologique semblent l'attester.

Nous pouvons envisager des pistes visant à généraliser ces résultats ponctuels.

Nous devons prendre en compte le principe piagétien des connaissances qui dérivent de l'action et dépasser les clivages théoriques mnésiques précités afin de valoriser différents types de mémoire qui sont complémentaires. Pour ce faire, nous devons mettre en avant :

- la mémoire de travail, les processus répétitifs et associatifs, la mémoire échoïque,

- l'ancrage perceptif et moteur,

- la prise en compte de l'affect et du système sensori-moteur, ainsi que de la mémoire épisodique,

- la mémoire implicite, procédurale et sémantique.

Retenons qu'une contextualisation facilitée par les nouvelles technologies, l'enseignant(e) et les accessoires ne peut que contribuer au décodage, encodage, assimilation, accommodation phonologique, et favoriser la récupération. Privilégier les conditions situationnelles, cognitives et affectives de la cognition sur le long terme ne pourra qu'activer mémoire de travail, mémoire épisodique, mémoire implicite procédurale et sémantique.

\section{Conclusion}

Compte tenu des enjeux liés à l'âge et à la réceptivité des enfants, la maîtrise de la composante phonologique est incontournable à l'école. Suite à une étude des types de mémoire, nous avons tenté de transcender le cloisonnement du spectre scientifique traditionnel. Nous avons insisté sur un ancrage perceptif et moteur, sur l'apport de la mémoire de travail se focalisant sur la répétition et les processus associatifs, sur la prise en compte de l'affect propre au public concerné, sur le système sensori-moteur et la mémoire "échoïque", sur la mémoire épisodique. Nous pouvons supposer que le processus d'appropriation phonologique peut être favorisé notamment par l'utilisation d'un cédérom adapté. Nous pouvons ainsi partager la remarque de P.Buser (1998: 298) qui constate "l'interaction entre les sphères cognitive et affective à tous les niveaux des rappels mnésiques". 
Nous pouvons conclure que "la mémorisation sera d'autant meilleure que l'information aura interpellé le cerveau tout entier, sous cortical et cortical, l'être affectif et cognitif" (Trocmé-Fabre, 1992 : 76). Une subtile alchimie entre l'enseignant et la machine nous semble prometteuse pour la mémorisation phonologique à très long terme, facilitant ainsi la continuité, non seulement de l'apprentissage de l'anglais, discipline linguistique, mais de l'apprentissage basé sur l'anglais, langue d'apprentissage des disciplines non linguistiques tout au long de la vie.

\section{BIBLIOGRAPHIE}

Andersen, M.(1983). Pidginization and creolization as language acquisition, New York: Newbury House.

Baddeley, A. (1992). La mémoire humaine, théorie et pratique, Grenoble : PUG.

Baddeley, A. (2000). "The episodic buffer: a new concept of working memory?" Trends in cognitive sciences, vol 4, $\mathrm{n}^{\circ} 11,417-423$.

Bailly, D. (1998). Les mots de la didactique des langues : le cas de l'anglais, Paris : Ophrys.

Beacco, J.C. (2007). L'approche par compétences dans l'enseignement des langues, Paris : Didier.

Buser, P. (1998). Cerveau de soi, cerveau de l'autre, Paris : Odile Jacob.

Cyrulnik, B. (1984). Mémoire de singe et parole d'homme, Paris : Hachette.

Delannoy, C. (1994). Une mémoire pour apprendre, Paris : Hachette.

Develay, M.(1993). "Les trois coups sont frappés : la représentation peut commencer", Cahiers pédagogiques, $\mathrm{n}^{\circ} 312$, p. 45.

Dörnyei, Z. (2001). Motivational strategies in the language classroom, Cambridge: CUP.

Ellis, R. (1994). The study of second language acquisition, Oxford: OUP.

Ellis, N.C. (2001). "Memory for language". In P. Robinson (dir.) Cognition and second language instruction, Cambridge: CUP. pp. 33-68.

Ginet, A. (1997). Du laboratoire de langues à la salle multimédias, Paris : Nathan

Goleman, D. (1997). L'intelligence émotionnelle, Paris : Laffont.

Laborit, H. (1994). La légende des comportements, Paris : Flammarion.

Laborit, H. (1996). Une vie, Paris : Éditions du Félin.

Linard, M. (1996). Des machines et des hommes, Paris : L'Harmattan.

Lieury, A. (1997). Mémoire et réussite scolaire, Paris : Dunod.

Martin-Cochet, (1999). O. Hello kids! Paris : Hachette multimédia.

Narcy-Combes, J-P. (2005). Didactique des langues et TIC, Paris : Ophrys. 
O’Neil, C. (1993). Les enfants et l'apprentissage des langues étrangères, Paris : Credif-HatierDidier.

Randall, M. (2007). Memory, psychology and second language learning, Philadephia: John Benjamins.

Roach, P. (2000). English phonetics and phonology, Cambridge: CUP.

Robinson, P. (2001). Cognition and second language instruction, Cambridge: CUP.

Singleton, D. (1989). Language acquisition: the age factor, Clevedon: Multilingual Matters.

Tagliante, C . (2005). L'évaluation et le Cadre européen commun de référence pour les langues, Paris : Clé International.

Thill, E. (1997). Motivation, émotion, attention et concentration, Paris : Vigot.

Tiberghien, G. (1991). Psychologie de la mémoire humaine, Grenoble : PUG.

Troubetzkoy, N .S. (1986). Principes de phonologie, Paris : Klinsieck.

Trocmé-Fabre, H. (1992). J'apprends, donc je suis, Paris : Éditions d'Organisation.

Tulving, E. (1983). Elements of episodic memory, Oxford: OUP.

Van Der Linden, M. \& Bruyer R. (1991). Neuropsychologie de la mémoire humaine, Grenoble :

PUG.

Williams, L.V. (1986). Deux cerveaux pour apprendre, Paris : Éditions d'Organisation.

\section{RÉSUMÉS}

Cette recherche action se veut qualitative, expérimentale, descriptive et analytique. Nous nous centrons sur l'apprentissage qui s'explique par la complexité du processus de mémorisation, tant au niveau de la dimension mnésique qu'au niveau didactique. Grâce à de nouveaux principes didactiques humanistes, nous analysons le problème de l'âge, les phases d'assimilation et d'accommodation phonologique, la mémoire de travail, l'affect, le système sensori-moteur, les processus associatifs pour mieux délimiter la mémoire épisodique. Nous mettons en œuvre une séquence privilégiant le décodage, l'encodage, la consolidation pour tester l'accommodation phonologique. Nous présentons une liste de principes à retenir.

This action research is qualitative, experimental, descriptive and analytical. Our learningcentred study takes into account a complex memorizing process based on a mnesic and didactic dimension. Thanks to new humanist didactic processes, we analyze the age factor, phonological assimilation and accommodation phases, working memory, the affective dimension, the sensory system and associative processes in order to scan episodic memory. We set up a sequence which focuses on decoding, encoding and consolidation to test phonological accommodation. We consequently present a list of principles to be followed.

\section{INDEX}

Mots-clés : mémorisation, phonologie, nouvelles technologies, processus humanistes Keywords : memorizing, phonology, new technologies, humanist processes 


\section{AUTEUR}

\section{YVON ROLLAND}

Yvon Rolland est Maître de conférence HDR au Laboratoire CRLHOI (Centre de Recherches Littéraires et Historiques de l'Océan Indien) EA 4078 de l'université de la Réunion, spécialiste de didactique de l'anglais.

Courriel : y.roll[at]orange.fr Adresse : Laboratoire CRLHOI Université de la Réunion, 15 Avenue René Cassin BP 715197715 Saint Denis cedex 9, France. 